\title{
6 \\ LA COLABORACIÓN DOCENTE COMO FACTOR DE APRENDIZAJE Y PROMOTOR DE MEJORA. UN ESTUDIO DE CASOS
}

\author{
(TEACHER COLLABORATION AS A FACTOR FOR LEARNING AND SCHOOL \\ IMPROVEMENT. A CASE STUDY)
}

Gabriela J. Krichesky

Universidad Torcuato Di Tella, Buenos Aires (Argentina)

F. Javier Murillo

Universidad Autónoma de Madrid

DOI: $10.5944 / e d u c X X 1.20181$

Cómo referenciar este artículo/How to reference this article:

Krichesky, G. J. y Murillo F. J. (2018). La colaboración docente como factor de aprendizaje y promotor de mejora. Un estudio de casos. Educación XX1, 21(1), 135-156, doi: 10.5944/educXX1.20181

Krichesky, G. J. \& Murillo F. J. (2018). La colaboración docente como factor de aprendizaje y promotor de mejora. Un estudio de casos. [Teacher collaboration as a factor for learning and school improvement. A case study]. Educación XX1, 21(1), 135-156, doi: 10.5944/educXX1.20181

\section{RESUMEN}

La investigación ha demostrado que la colaboración docente es una condición esencial para impulsar procesos de innovación y mejora en los centros educativos. Sin embargo no todo trabajo colaborativo estimula mejoras sustanciales en la enseñanza o desarrolla la capacidad de innovación del profesorado. Por ello esta investigación pretende describir y comprender cuáles son las prácticas colaborativas que pueden potenciar el aprendizaje del profesorado, generando así mayor capacidad colectiva para implementar procesos de innovación y mejora escolar. Dada la naturaleza cualitativa de nuestra indagación, optamos por un estudio de casos instrumental y de corte etnográfico en el que se analizan en profundidad dos institutos de educación secundaria caracterizados por una acentuada cultura de trabajo colectivo y procesos de innovación exitosos. Los datos se recogieron a través de entrevistas semi-estructuradas, análisis de documentación y observaciones participantes y no participantes. Se encontró que la colaboración docente puede manifestarse a través de prácticas ligadas a la coordinación, el desarrollo conjunto y la resolución de problemas. La coordinación se asoma como una de las modalidades más débiles de trabajo colaborativo: no requiere de valores compartidos, no genera sólidas relaciones de interdependencia y no fomenta 
necesariamente el aprendizaje docente. El desarrollo de proyectos interdisciplinarios y la resolución conjunta de problemas, en cambio, demandan una fuerte interdependencia sobre la base de valores compartidos y se asientan en intercambios con una gran potencialidad para generar nuevos aprendizajes. La investigación sobre la colaboración docente resulta estratégica de cara al cambio en educación: entender qué hacen y de qué hablan los profesores cuando se reúnen y qué impacto tienen estos intercambios en su capacidad de aprendizaje permitirá sin dudas profundizar nuestro conocimiento sobre la innovación educativa y la mejora escolar.

\section{PALABRAS CLAVE}

Colaboración docente; mejora escolar; innovación educativa; desarrollo profesional.

\section{ABSTRACT}

Research has shown that teacher collaboration is essential for boosting educational innovation and school improvement. However, not all collaborative practices can stimulate substantial changes in teaching or develop the capacity for innovation. Therefore, this study aims to describe and comprehend which collaborative practices can potentially have an impact on teacher learning while encouraging collective capacity to develop educational innovation and improvement processes. Given the qualitative nature of our inquiry on collaborative work, we conducted an instrumental and ethnographic case study in which we analyzed two high schools characterized by a collaborative culture with satisfactory innovative processes. Data was collected through semi-structured interviews, documentation analysis and participant and nonparticipant observations. Our findings suggest that teacher collaboration can occur through different practices related to coordination, joint development and problem solving. Coordination appears as one of the weakest forms of collaborative work: it does not require shared values, no interdependent relationships are generated and it does not necessarily encourage teacher learning. Instead, the development of interdisciplinary projects and joint problem solving demands strong teacher interdependence on the basis of shared values, while offering exchanges with great potential to generate teacher learning. Research on teacher collaboration therefore appears strategic towards educational change: we need to comprehend what teachers do and what they talk about when they meet, and the impact these exchanges have on their ability to learn and their capacity to change. This will undoubtedly deepen our knowledge on educational innovation and school improvement.

\section{KEY WORDS}

Teacher collaboration; school improvement; educational innovation; professional development. 


\section{INTRODUCCIÓN}

La investigación ha demostrado que la colaboración entre docentes es una condición esencial para impulsar procesos de innovación y mejora en los centros educativos (Donmoyer, Yennie-Donmoyer y Galloway, 2012; Sebastian y Allensworth, 2012; Vescio, Ross y Adams, 2008). Sin embargo, no todas las prácticas colaborativas estimulan mejoras sustanciales en la enseñanza o desarrollan la capacidad de innovación del profesorado. El trabajo colaborativo entre profesores, según su naturaleza, puede impulsar estrategias de enseñanza innovadoras o perpetuar prácticas pedagógicas conservadoras (Little y Horn, 2007).

Reconocer cuáles son las modalidades de trabajo colectivo que impulsan la innovación y qué tipo de intercambios promueven la capacidad de mejora del profesorado se ha tornado en un objetivo primordial para la investigación educativa. Este artículo recoge los principales resultados de un estudio que busca describir y comprender en qué medida el trabajo colaborativo puede incidir en el desarrollo de la innovación y la mejora escolar, a través de una investigación etnográfica en dos institutos de educación secundaria de la Comunidad de Madrid.

\section{MARCO TEÓRICO}

En los últimos años se han diversificado considerablemente las plataformas ideológicas desde las que se ha explorado la idea de la colaboración docente (Lavié Martínez, 2004a). Así, conviven enfoques críticos que denuncian la utilización de la colegialidad como mecanismo de control (Smyth, 2001), con perspectivas más alentadoras que asumen a la colaboración como factor de cambio educativo (Hargreaves y Shirley, 2012), como componente clave del aprendizaje organizacional (Gairín, 2000; Senge, 2002) y como estrategia de mejora de los centros escolares (McLaughlin y Talbert, 2006).

Si bien las investigaciones son hoy más moderadas en cuanto a sus expectativas, lo cierto es que continúa prevaleciendo un tono más bien normativo que prescribe a la colegialidad como imperativo, a veces moral y otras simplemente técnico, para comandar el trabajo docente (Lavié Martínez, 2006). Sin embargo, sabemos que la colaboración puede supeditarse a distintos paradigmas y propósitos en torno a la escolarización, el trabajo docente y la reforma educativa, por tanto resulta clave identificar las perspectivas que definen la identidad de los intercambios entre el profesorado (Escudero, 2009). 
Lo primero que hemos aprendido acerca de la colaboración docente es lo arduo que resulta «desarrollarla» en las escuelas (Little, 1990; Rosenholtz, 1989), ya sea por las condiciones físicas y estructurales de las organizaciones escolares (Lortie, 1975), por la falta de oportunidades formativas que contribuyan al desarrollo de destrezas vinculadas al trabajo colaborativo (Day, 2005) o por la limitada capacidad para gestionar posiciones divergentes (Grossman, Wineburg y Woolworth, 2001). En efecto Huberman (1993) indica que la ausencia de estructuras institucionales para efectivamente resolver problemas y repensar conjuntamente la práctica, desalienta al profesorado a implicarse en actividades colaborativas puesto que son percibidas como una distracción de las necesidades más apremiantes de sus alumnos. Así, la interacción con colegas se valora como positiva solo en cuanto se torna relevante para satisfacer las urgencias que demanda el aula (Lavié Martínez, 2004b).

En este trabajo asumimos que, bajo ciertas condiciones, la colaboración puede constituirse en una alternativa de trabajo atractiva para el colectivo docente y una estrategia interesante de mejora. Adoptamos así a la línea de investigación sobre Mejora Escolar como marco para comprender precisamente cuáles son esas condiciones y de qué manera la colaboración puede resultar estratégica para el profesorado en tanto factor de aprendizaje y motor para la innovación escolar.

Numerosos estudios han demostrado que la colaboración habilita nuevas oportunidades de desarrollo profesional basadas en la reflexión compartida con colegas sobre los dilemas y problemas de la práctica (Butler y Schnellert, 2012; Horn y Little, 2010). Desde esta perspectiva, los profesores aprenden conjuntamente a partir de procesos de indagación colectiva basados en el análisis de evidencias (Duke, 2006) y la observación de clases (Hopkins, 2010). Se procura identificar las necesidades de aprendizaje del alumnado para, a partir de ellas, establecer las necesidades de aprendizaje de los propios docentes y diseñar espacios de formación acordes a estas (Hord y Hirsch, 2008). La colaboración se constituye así en una herramienta para la mejora de la enseñanza dado que los intercambios proveen los recursos para aprender en y desde la práctica, alentando diálogos fundamentados en la experiencia de clase (Jäppinen, Leclerc y Tubin, 2015; Little, 2002; Martin, 2014; Silins y Mulford, 2004).

La colegialidad puede asimismo estimular la motivación y el compromiso del profesorado en la medida en que se consensúen los propósitos de la colaboración (DuFour, DuFour, Eaker y Many, 2010) en un clima de altas expectativas sobre el trabajo docente y los progresos del alumnado (Goddard, Hoy y Hoy, 2000). De esa manera, el trabajo colegiado brinda una plataforma para generar una visión compartida que oriente la toma de 
decisiones a favor de los estudiantes y en congruencia con las posibilidades de los docentes.

Cabe destacar que el rol de los directivos resulta clave a la hora de garantizar las condiciones necesarias para generar ambientes de trabajo colaborativo (Szczesiul, 2014; Szczesiul y Huizenga, 2014). Es el equipo directivo el que se ocupa de que los intercambios conserven un foco constante en los aprendizajes de los estudiantes (Bolam, McMahon, Stoll, Thomas y Wallace, 2005), facilitando el acceso a recursos que estimulen el aprendizaje docente y garantizando la periodicidad de los encuentros (King y Bouchard, 2011; Muijs y Rumyantseva, 2014) en un entorno de confianza y respeto mutuo (Crawford, 2012; Huffman y Hipp, 2003; Thoonen, Sleegers, Oort y Peetsma, 2012).

\section{METODOLOGÍA}

El objetivo de la investigación consiste en describir y comprender de qué forma las prácticas colaborativas impactan en el aprendizaje del profesorado y alientan la capacidad colectiva por implementar procesos de innovación y mejora escolar.

Dada la naturaleza cualitativa de nuestra indagación se opta por un estudio de casos instrumental y de corte etnográfico en el que se analizan en profundidad dos institutos de educación secundaria durante todo el ciclo escolar 2012/2013. Estos institutos fueron seleccionados en el marco de un $\mathrm{I}+\mathrm{D}+\mathrm{i}$ financiado por el Ministerio de Ciencia e Innovación de España (EDU2010-18224) y un Proyecto de Investigación financiado por la Universidad Autónoma de Madrid (CCG10-UAM/HUM-5583), respectivamente. Tanto los investigadores que condujeron dichos estudios como orientadores e inspectores entrevistados destacaron a estas dos escuelas por sus culturas colaborativas. Para comprobarlo, mantuvimos un primer encuentro con miembros de cada equipo directivo en el que efectivamente constatamos que los centros satisfacían los criterios señalados por la literatura: en ambas escuelas se establecen espacios periódicos de encuentro entre docentes destinados a atender a las necesidades del alumnado, en los que se alienta la experimentación y la reflexión colectiva.

Los dos institutos seleccionados, a los que atribuimos denominaciones ficticias, han ganado importantes distinciones en materia de innovación escolar. Uno de ellos ha recibido numerosos premios de mérito en Bachillerato, ha ganado el concurso sobre Rutas Científicas, ha participado en congresos para exponer propuestas pioneras sobre diversificación escolar y ha publicado diversos artículos sobre la enseñanza de las ciencias naturales. 
El otro fue el primer instituto en adelantar la implantación de la Educación Secundaria Obligatoria (ESO) y recibió numerosos premios gracias a su Programa de Mediación y Tratamiento de Conflictos. Es asimismo uno de los Centros de Innovación Tecnológica de la Comunidad de Madrid y uno de los primeros en recibir dicha denominación. A su vez las escuelas han mejorado, año a año, su desempeño en las Pruebas de Conocimientos y Destrezas Indispensables (CDI) implementadas por la Comunidad de Madrid, con un avance notable en el período 2011-2013.

Los datos se recogieron a través de entrevistas semi-estructuradas, análisis de documentación y observaciones participantes y no participantes. El trabajo de campo se prolongó un total de nueve meses e inició con un primer contacto con miembros del equipo directivo de cada centro. Se procedió luego con el análisis de los documentos más relevantes como ser el Proyecto Educativo de Centro, el Plan de Convivencia, la Programación General Anual y diferentes actas de reuniones docentes, de departamento y de la Comisión de Coordinación Pedagógica (CCP), con el fin de identificar los principales contenidos y acuerdos alcanzados en distintas instancias de trabajo colegiado. Se realizaron entrevistas semi-estructuradas a todos los miembros del equipo directivo, a todos los jefes de departamento y algunos profesores considerados «clave» en las que se procuró atender a las siguientes interrogantes, entre otras:

- ¿Qué metas u objetivos persigue el trabajo en equipo?

- ¿Qué modalidades y formatos adopta la colaboración docente?

- ¿Existen variaciones en la colaboración docente según áreas, niveles y/o departamentos?

- ¿Cuáles son los principales contenidos de las reuniones en equipo? ¿Quién y cómo se definen?

- ¿Cuáles son las principales ventajas de la colaboración percibidas por el profesorado en esta escuela?

- ¿Qué incidencia tiene la colaboración en el aprendizaje docente y en la mejora escolar?

Se realizaron un total de 46 entrevistas individuales y 12 grupos de discusión con coordinadores, profesores y alumnos, respectivamente. En paralelo se desarrollaron también, con una frecuencia quincenal, observaciones participantes y no participantes de situaciones que considerábamos relevantes para nuestro estudio, como las reuniones de departamento, reuniones de claustro, seminarios de formación docente y actividades especiales. El análisis de los datos se desarrolló durante nuestra «estancia en el escenario» siguiendo los principios y procedimientos de la Teoría Fundamentada (Glaser y Strauss, 1967). 


\section{RESULTADOS}

El IES Las Alondras está situado en una gran ciudad residencial al sur de la Comunidad de Madrid. Es el de menor tamaño del barrio y el más reciente: fue creado a finales de los años 80 como respuesta ante la llegada de nuevos inmigrantes, sumado a un importante aumento de natalidad (tabla 1). Se constituye actualmente como un Centro de Atención Prioritaria por la cantidad de alumnos inmigrantes, con un programa de diversificación especialmente valorado por la inspección escolar. El IES Los Girasoles, por su parte, tiene más de 30 años de antigüedad y está ubicado en una zona residencial de clase media al sudoeste de la Comunidad de Madrid. Cuenta con más de 50 profesores y una matrícula compuesta por 687 estudiantes. Fue el primer instituto en adelantar la implantación de la Educación Secundaria Obligatoria (ESO) en su ciudad y recibió numerosos premios gracias a su Programa de Mediación y Tratamiento de Conflictos. Es asimismo uno de los Centros de Innovación Tecnológica de la Comunidad de Madrid y por todo ello cuenta con un gran reconocimiento en la zona.

Tabla 1

Datos del IES Las Alondras y del IES Los Girasoles. Curso escolar 2012-2013

\begin{tabular}{|c|c|c|}
\hline & IES LAS ALONDRAS & IES LOS GIRASOLES \\
\hline Antigüedad del centro & 24 años & 33 años \\
\hline Cantidad de alumnos & $\begin{array}{c}313 \text { (44\% de población } \\
\text { inmigrante) }\end{array}$ & $\begin{array}{c}687 \text { (10,6\% de población } \\
\text { inmigrante })\end{array}$ \\
\hline Cantidad de grupos & $\begin{array}{l}12 \text { grupos y medio (por } \\
\text { desdobles en Bachillerato) }\end{array}$ & 24 grupos \\
\hline Líneas & 2 & 5 \\
\hline Cantidad de profesores: & 36 docentes & 51 docentes \\
\hline Ofertas de Bachillerato: & $\begin{array}{c}\text { Ciencias y Tecnología, } \\
\text { Humanidades y Ciencias } \\
\text { Sociales }\end{array}$ & $\begin{array}{l}\text { Humanidades y Ciencias } \\
\text { Sociales, Ciencias de la } \\
\text { Naturaleza y de la Salud }\end{array}$ \\
\hline
\end{tabular}

Fuente: Elaboración propia.

\section{IES Las Alondras}

Los profesores y profesoras del IES Las Alondras consideran que la colaboración atiende esencialmente a dos propósitos. En primer lugar incide en el desarrollo de un buen ambiente de trabajo. La directora explica que 
la colaboración "genera un sentimiento de unidad en el centro y promueve relaciones más satisfactorias entre los docentes». Por otra parte, la colaboración está al servicio de la resolución de conflictos y por ello es que «la intervención ante problemas concretos es siempre una intervención colectiva» (Profesor 1). El trabajo colegiado aparece entonces como una herramienta esencial para mantener un clima escolar adecuado.

En ese centro la colaboración se cristaliza principalmente en labores vinculadas a la coordinación. En efecto, en repetidas ocasiones los profesionales de esta escuela asociaron la colaboración con el concepto de coordinación:

«En los departamentos se colabora mucho. Intentamos coordinarnos para que los exámenes sean iguales y luego intentamos hacer ejercicios muy similares. Intentamos ir todos a la vez en la programación y tener los mismos criterios de calificación; seguimos pautas comunes de trabajo» (Directora).

En este contexto, la coordinación se asume como una estrategia especialmente relevante que permite alcanzar acuerdos y establecer pautas de trabajo comunes entre los profesores. Se asume que esta coordinación consolida el desarrollo de una línea de trabajo común y beneficia al alumnado por medio de la adopción de criterios compartidos sobre la enseñanza y la evaluación. Así, una profesora explica que «nos ponemos de acuerdo incluso en cómo dar determinados temas. Decidimos que si la regla de tres se hace de esta manera, lo hacemos todos igual para que al año siguiente no les cueste a los alumnos» (Profesor 2).

Bajo el firme convencimiento de que esto beneficia a los estudiantes, la coordinación entre el profesorado permite alcanzar determinados acuerdos en materia de secuenciación, organización y programación de los contenidos. Aparece así como una de las principales atribuciones que se adjudican al trabajo colaborativo en este centro. La propia Jefa de Estudios enfatiza esta acepción alegando que "no es tan difícil (lograr) la colaboración. Ponerse de acuerdo con alguien no es difícil». Esto demuestra que incluso a menudo los conceptos se confunden: colaboración y coordinación aparecen como sinónimos.

La colaboración docente se hace además patente a través del desarrollo de proyectos interdisciplinarios, sobre todo en el área de las ciencias naturales. Las Rutas Científicas y el Proyecto GLOBE (Programa de Aprendizaje y Observaciones Globales en Beneficio del Medio Ambiente) son muestra de ello. En ambos participan profesores de Biología, Geología, Física, Química y Tecnología. Algunos docentes reconocen que este trabajo 
se ve favorecido por el tamaño reducido del centro ya que esto permitió unificar las reuniones de departamento para poder trabajar de manera conjunta e interdisciplinaria.

Entre las principales condiciones que facilitan la colaboración en el centro, los profesores destacan el nivel de comunicación: alegan que la información circula de manera constante y sistemática. Agradecen que desde el equipo directivo se comparta información relevante sobre cuestiones que hacen al funcionamiento del instituto. Las reuniones de Comisión de Coordinación Pedagógica, así como también las de departamento, se conciben como los canales de comunicación por excelencia para que la información fluya de abajo-arriba y de arriba-abajo.

Por otra parte, en esta escuela se proveen espacios y tiempos específicos que facilitan el encuentro entre docentes. Existe una gran cantidad de reuniones que se realizan fuera del horario escolar, más allá de las juntas de evaluación establecidas. De hecho, los profesores de esta escuela consideran que se reúnen más que en otros institutos, sobre todo "para tomar decisiones con respecto a la disciplina» (Profesor 4). A menudo estos espacios se generan por iniciativa del propio cuerpo docente, lo cual denota una importante predisposición hacia el trabajo colectivo: «siempre hay mucha gente dispuesta a reunirse y trabajar en los proyectos que nos proponen» (Profesor 5).

La colaboración entre los profesores se configura entonces como uno de los elementos más representativos del IES Las Alondras. El profesorado entiende que dicho trabajo colaborativo permite resolver los conflictos de manera más eficaz, lo cual contribuye también al desarrollo de un buen clima de trabajo tanto entre profesores como entre los alumnos. Los docentes perciben también que la colaboración mejora la práctica en tanto permite coordinar las acciones pedagógicas, lo cual genera una línea de trabajo común en beneficio de los estudiantes. El diseño e implementación de proyectos conjuntos y/o interdisciplinarios es también, aunque en menor grado, otra de las facetas que adopta la colaboración en este centro.

\section{IES Los Girasoles}

La colaboración en el IES Los Girasoles tiene matices diferentes. El trabajo colegiado está explícita y directamente orientado hacia la mejora de la enseñanza y el aprendizaje. Hace unos años el equipo directivo diseñó el proyecto «Todos a Una» mediante el cual se procuró fortalecer el trabajo en equipo alentando a la construcción de acuerdos compartidos 
para edificar una línea de trabajo común. Se impulsó además el desarrollo de propuestas interdisciplinarias en beneficio del aprendizaje de los estudiantes. Ejemplo de ello es el proyecto Comenius, que promueve la movilidad y la cooperación entre centros educativos europeos a través de proyectos de intercambio, donde la idea es «dinamizar el trabajo colectivo y potenciar las actividades en conjunto del profesorado para que las propuestas formativas sean más interesantes para los estudiantes» (Profesor 1).

A su vez el trabajo colaborativo se ha profundizado en virtud de haberse constituido como Instituto de Innovación Tecnológica. Los docentes implicados admiten que dicho proyecto permite «acceder, compartir y utilizar los recursos de otros colegas» (Profesor 2) lo cual ha supuesto el intercambio de materiales de manera recurrente. En referencia a ello una profesora comentaba que la buena disposición hacia el uso compartido de los recursos sucede porque los docentes no son particularmente celosos de sus productos y experiencias.

La colaboración se manifiesta también a través de otras prácticas: (a) el desarrollo de materiales, (b) la coordinación, (c) el trabajo interdisciplinario, y (d) la planificación conjunta de actividades generales. En cuanto al desarrollo de materiales, si bien las plataformas virtuales contribuyen a que esto suceda con frecuencia, lo cierto es que la elaboración conjunta de recursos pedagógicos se realiza hace ya varios años. Los cuadernillos sobre mediación escolar generados para los docentes ingresantes o los documentos de trabajo elaborados por profesores del departamento de Matemáticas son ejemplo de ello.

La coordinación aparece como otro ejemplo de colaboración y es asumida como un mecanismo para establecer plazos o acuerdos entre los profesores enfocados, generalmente, a la temporalización de los contenidos. En este sentido, las reuniones de departamento se suelen dedicar a «la coordinación de contenidos: saber por dónde van los demás, qué están dando» (Profesor 3).

El trabajo colaborativo se hace también presente en el desarrollo conjunto de actividades interdisciplinarias. Los docentes programan de forma colectiva para luego implementar propuestas que conjuguen contenidos de dos o más asignaturas. Ejemplo de ello son una serie de clases diseñadas por un docente de Matemáticas y uno de Educación Física en el que se pretende que los alumnos utilicen los resultados de sus propias pruebas físicas para luego desarrollar ejercicios de análisis estadístico. En otra ocasión, docentes del área de Lengua y Tecnología planificaron un proyecto denominado Libromatón, en el que los estudiantes deben articular conocimientos 
de ambas asignaturas para generar materiales audiovisuales sobre autores literarios.

Por último, la colaboración se evidencia en el diseño e implementación de las actividades generales del centro en las que están implicados la mayoría de los profesores. La directora considera que el evento más trascendente del año, la «Jornada de 24 horas», es uno de los mejores ejemplos del trabajo en equipo porque «te puedes encontrar a las dos de la mañana con 25 profesores dispuestos a colaborar para que el evento salga bien».

Entre las condiciones que facilitan y promueven el trabajo en equipo en este instituto los profesores distinguen: los tiempos y espacios destinados a reuniones docentes, la estabilidad del plantel y los cursos de formación en el centro. Generar espacios y tiempos para que los profesores puedan dedicarse a trabajar colectivamente resulta primordial y para ello los horarios se flexibilizan: los jefes de estudio están siempre dispuestos a reorganizar las guardias o incluso a reemplazar profesores para facilitar una reunión o el desarrollo de alguna actividad conjunta.

En segundo lugar se considera que la estabilidad de los profesores ha sido fundamental. Una profesora señala que el hecho de no contar con docentes interinos evita tener que volver a programar y a consensuar decisiones todos los años. Así explica que la estabilidad permite «que te puedas plantear, como equipo, algo realmente serio».

Por otra parte el desarrollo de grupos de trabajo y seminarios como espacios de formación en el centro también ha favorecido la colaboración entre el profesorado. Este instituto es reconocido en la zona por la calidad y cantidad de cursos de formación internos que se dictan en horario extracurricular, a tal punto que docentes de escuelas vecinas asisten a los mismos con recurrencia. Estos cursos suelen dictarse por miembros del claustro y surgen a partir de la detección de necesidades en el propio centro. Ejemplo de ello son las instancias de actualización sobre estrategias de enseñanza en el área de las matemáticas o los cursos sobre mediación escolar.

La colaboración en este centro es entonces obra y consecuencia de una serie de acciones cuidadosamente diseñadas para tal fin. Esto ha permitido construir una base para el desarrollo de innovaciones ligadas a la mejora de la convivencia y la implementación de nuevas tecnologías, a la vez que unificar ciertos criterios de actuación en la enseñanza de cada asignatura. 


\section{ANÁLISIS Y DISCUSIONES}

En los dos institutos se perciben altos niveles de colaboración entre sus miembros y en ambos casos resulta clave el clima de apoyo, respeto y confianza que impera entre el profesorado (McLaughlin y Talbert, 2006), así como también la predisposición de los directivos a facilitar los encuentros entre colegas (Huffman y Hipp, 2003). Sin embargo, entre los centros hay una diferencia esencial en la manera de concebir y concretar dicho trabajo colectivo.

\section{Los sentidos de la colaboración}

En el IES Las Alondras la colaboración se asocia principalmente al mantenimiento de un buen ambiente, tanto en lo referido al clima de trabajo como al clima de aula y aprendizaje. La mayoría de las acciones colaborativas en esta escuela están al servicio de garantizar una vida escolar armoniosa, coherente y calma. Al interactuar con los colegas, los docentes no buscan necesariamente modificar sus prácticas, discutir patrones de actuación o anticiparse a procesos de cambio: en esta escuela la colaboración resuelve, estabiliza, ordena. En el IES Los Girasoles, en cambio, la colaboración se ha asumido como una estrategia para modificar la forma en la que se llevan a cabo ciertos procesos en la escuela y, por tanto, ha sido objeto de una política explícita para su desarrollo. Así, mientras que en un caso la colaboración se asume como una práctica inherente a la forma de trabajo del profesorado, perpetuada por los propios docentes, en el otro el trabajo colectivo es fruto de una serie de acciones diseñadas para tal fin.

Aparecen entonces dos concepciones bien distintas: la colaboración como política de trabajo y la colaboración como estrategia de cambio. En el primer caso y coincidiendo con las experiencias registradas en el IES Las Alondras, la colaboración caracteriza las relaciones interpersonales y los lazos profesionales entre docentes, lo cual permite (y esto no es poco) mantener un buen clima escolar en el que se pueda desarrollar eficazmente la tarea docente. Esto favorece la coherencia en la enseñanza, facilita la adopción de criterios comunes, optimiza la resolución de conflictos y promueve una mayor satisfacción docente.

La colaboración como estrategia de cambio supone estimular interacciones dirigidas específicamente a generar alteraciones. En el IES Los Girasoles la colaboración entre el profesorado se aborda como medio para dar respuesta a ciertas problemáticas: la falta de motivación del alumnado, la fragmentación de la práctica docente, los problemas de convivencia. La colegialidad aparece como una estrategia para modificar la experiencia escolar 
y esto obliga a clarificar previamente los objetivos y contenidos de dicha colaboración. Esto conforma un terreno fértil para generar intercambios que habiliten el diseño de soluciones y propuestas en respuesta a problemas escolares específicos. Así la colaboración adopta un matiz estratégico que responde a las necesidades de mejora de la escuela (Bolam y otros, 2005; Horn y Little, 2010).

\section{Modalidades de trabajo colaborativo}

De acuerdo a los fines a los que está subsumido el trabajo conjunto entre los docentes, la colaboración puede adoptar distintos matices y concretarse a través de diferentes prácticas: (a) la coordinación, (b) el desarrollo conjunto y (c) la resolución de problemas. Se abordará cada una de estas clasificaciones procurando atender a las siguientes dimensiones:

- Hasta qué punto dicha colaboración promueve o se sostiene sobre la base de relaciones de trabajo interdependientes.

- Hasta qué punto implica compartir una visión o valores comunes.

- Hasta qué punto supone un aprendizaje para los profesores implicados.

\section{a) La coordinación}

Para comprender el sentido que se le otorga al concepto de «coordinación» bien valdría recuperar la idea de coordenada. Una coordenada indica una posición, permite emitir una referencia con respecto a una localización específica: es una forma de dar a conocer una ubicación. Cuando los docentes de ambos institutos hacen referencia a su trabajo de coordinación aluden precisamente a esta acepción. Coordinarse consiste en compartir una ubicación o posición con los colegas en términos de programación, supone informar y estar informado sobre los temas que se están abordando.

Aun cuando la coordinación permite también alcanzar acuerdos y decisiones respecto a la secuenciación de contenidos, las fechas de exámenes o incluso las estrategias a desarrollar dentro del aula, esta no genera ni potencia, necesariamente, vínculos interdependientes sólidos. Los profesores continúan realizando su labor de forma independiente, respetando acuerdos y plazos consensuados, sin que esto incite a trabajar con colegas con mayor frecuencia o profundidad. La coordinación ofrece así pautas para la acción individual sin que ello genere un trabajo verdaderamente articulado con otros. 
Para coordinarse no es necesario que los docentes compartan valores o visiones comunes sobre la enseñanza. De hecho, la coordinación no promueve el aprendizaje del profesorado dado que, al no basarse forzosamente en discusiones pedagógicas profundas, la información compartida o los acuerdos adoptados no impactan en la forma de pensar y proceder de los docentes. En sintonía con el enfoque de Little (1990) la coordinación puede resultar importante pero de ninguna manera suficiente para generar intercambios que tengan un impacto sustancial en la tarea docente.

\section{b) El desarrollo conjunto}

La colaboración puede suponer también la conjunción de esfuerzos para generar un producto final que puede ser un recurso, un proyecto interdisciplinario o una actividad general. Desarrollar materiales didácticos supone un trabajo colectivo a merced de obtener un recurso para lo cual cada implicado aporta conocimientos, experiencias u otros materiales. Generalmente esto requiere de una instancia previa para discutir y establecer objetivos, contenidos, etc., y así desarrollar enfoques compartidos: ¿A qué necesidades responde dicho recurso? ¿En qué sentido facilita el aprendizaje? ¿Cómo puede apoyar la enseñanza?

El desarrollo conjunto de recursos puede impulsar el aprendizaje docente dado que el intercambio de visiones, materiales y conocimientos puede estimular la adopción de nuevas prácticas. La interdependencia entre docentes es asimismo un poco más marcada dado que, aun cuando elaborar un material implique aglutinar distintos aportes individuales, se necesita del esfuerzo de todos los contribuyentes: estas aportaciones solo cobran sentido en un marco general que les trasciende individualmente.

El desarrollo de proyectos interdisciplinarios supone relaciones de interdependencia fuertes, cada profesor necesita indefectiblemente del otro para el diseño y desenvolvimiento de la propuesta. La interacción entre ellos puede además estimular nuevos aprendizajes a raíz de aquello que se teje conjunta e imbricadamente. Un profesor de Educación Física reconoció por ejemplo que aprendió muchísimo cuando desarrolló zancos con una profesora de Tecnología a partir de la metodología de enseñanza que aplicaba su colega.

El desarrollo de actividades generales implica una conjunción de esfuerzos para eventos de dimensiones importantes en los que pueden coexistir diferentes formas de contribuir. Estos eventos se sostienen sobre la base de responsabilidades distribuidas y esto conlleva una interdependencia só- 
lida entre los implicados. Cuando la participación es voluntaria se presume que los profesores deciden colaborar porque en cierta forma valoran la naturaleza de la propuesta. Sin embargo, definir si el desarrollo conjunto de actividades generales genera o no aprendizajes resulta un tanto complejo. En algunos casos, los docentes se limitan a realizar tareas operativas, en otras ocasiones están implicados en instancias de diseño y planificación conjuntas. Dependerá por tanto de la función que asume cada miembro en el proceso y su interrelación con colegas para determinar si dicha participación estimula nuevos aprendizajes.

\section{c) La resolución de problemas}

Se trata en este caso de encontrar posibles soluciones a una situación percibida como problemática. Para ello los espacios de discusión permiten evaluar alternativas, debatir una decisión ya adoptada o planificar intervenciones colectivas. Es esencial que entre los implicados se compartan ciertos valores que transformen un determinado evento en algo problemático o, en todo caso, se edifique una visión común que oriente el análisis y la búsqueda de soluciones. A veces estas emergen cuando los profesores recuperan experiencias previas, buscan información o deciden incluso capacitarse para solventar el problema. En cualquier caso, las prácticas que se comparten o los conocimientos que se construyen generan un cuerpo de información que sirve de insumo de aprendizaje para el profesorado. La interdependencia es también sólida, tanto por la necesidad de escuchar distintas ideas para encontrar la alternativa óptima como para implementar intervenciones conjuntas (tabla 2).

Tabla 2

Caracterización de las distintas prácticas colaborativas

\begin{tabular}{|c|c|c|c|c|c|}
\hline & \multirow[b]{2}{*}{ Coordinación } & \multicolumn{3}{|c|}{ Desarrollo conjunto } & \multirow[b]{2}{*}{$\begin{array}{c}\text { Resolución } \\
\text { conjunta de } \\
\text { problemas }\end{array}$} \\
\hline & & $\begin{array}{c}\text { Desarrollo } \\
\text { conjunto de } \\
\text { materiales }\end{array}$ & $\begin{array}{c}\text { Desarrollo } \\
\text { conjunto de } \\
\text { actividades } \\
\text { interdiscipli- } \\
\text { narias }\end{array}$ & $\begin{array}{c}\text { Desarrollo } \\
\text { conjunto de } \\
\text { actividades } \\
\text { generales }\end{array}$ & \\
\hline $\begin{array}{l}\text { Objeto de la } \\
\text { colaboración }\end{array}$ & $\begin{array}{l}\text { Alcanzar } \\
\text { acuerdos, } \\
\text { tomar deci- } \\
\text { siones. }\end{array}$ & $\begin{array}{l}\text { Generar un } \\
\text { producto con- } \\
\text { creto }\end{array}$ & $\begin{array}{l}\text { Diseñar y } \\
\text { desarrollar } \\
\text { una actividad } \\
\text { o clase. }\end{array}$ & $\begin{array}{l}\text { Diseñar y } \\
\text { desarrollar } \\
\text { una actividad } \\
\text { general }\end{array}$ & $\begin{array}{l}\text { Generar alter- } \\
\text { nativas a una } \\
\text { situación per- } \\
\text { cibida como } \\
\text { problemática. }\end{array}$ \\
\hline
\end{tabular}




\begin{tabular}{|c|c|c|c|c|c|}
\hline & \multirow[b]{2}{*}{ Coordinación } & \multicolumn{3}{|c|}{ Desarrollo conjunto } & \multirow[b]{2}{*}{$\begin{array}{l}\text { Resolución } \\
\text { conjunta de } \\
\text { problemas }\end{array}$} \\
\hline & & $\begin{array}{c}\text { Desarrollo } \\
\text { conjunto de } \\
\text { materiales }\end{array}$ & $\begin{array}{c}\text { Desarrollo } \\
\text { conjunto de } \\
\text { actividades } \\
\text { interdiscipli- } \\
\text { narias }\end{array}$ & $\begin{array}{c}\text { Desarrollo } \\
\text { conjunto de } \\
\text { actividades } \\
\text { generales }\end{array}$ & \\
\hline $\begin{array}{l}\text { Valores com- } \\
\text { partidos }\end{array}$ & No necesarios & $\begin{array}{l}\text { Necesarios y } \\
\text { explícitos }\end{array}$ & $\begin{array}{l}\text { Necesarios, } \\
\text { pueden ser } \\
\text { implícitos o } \\
\text { explícitos }\end{array}$ & $\begin{array}{l}\text { Necesarios e } \\
\text { implícitos. }\end{array}$ & $\begin{array}{l}\text { Necesarios y } \\
\text { explícitos }\end{array}$ \\
\hline $\begin{array}{l}\text { Relaciones } \\
\text { interdepen- } \\
\text { dientes }\end{array}$ & Débiles & Moderadas & Sólidas & Moderadas & Sólidas \\
\hline $\begin{array}{l}\text { Aprendizajes } \\
\text { a partir de los } \\
\text { intercambios }\end{array}$ & Escasos. & $\begin{array}{l}\text { Amplios. } \\
\text { Se generan } \\
\text { a partir de } \\
\text { recursos y } \\
\text { experiencias } \\
\text { de otros do- } \\
\text { centes. }\end{array}$ & $\begin{array}{l}\text { Suficientes. } \\
\text { Se aprenden } \\
\text { contenidos } \\
\text { de otras } \\
\text { asignaturas, y } \\
\text { posiblemen- } \\
\text { te algunas } \\
\text { metodologías } \\
\text { didácticas. }\end{array}$ & $\begin{array}{l}\text { Variables. } \\
\text { Pueden darse } \\
\text { a partir de la } \\
\text { observación } \\
\text { de otras prác- } \\
\text { ticas. }\end{array}$ & $\begin{array}{l}\text { Amplios. Se } \\
\text { generan a } \\
\text { través de un } \\
\text { conjunto de } \\
\text { conocimientos } \\
\text { construidos } \\
\text { de forma con- } \\
\text { junta. }\end{array}$ \\
\hline
\end{tabular}

Fuente: Elaboración propia

En los dos institutos estudiados la coordinación aparece como una de las prácticas más asociadas al trabajo colaborativo pero con un impacto poco sustancial en los valores y las prácticas de enseñanza del profesorado. Mientras que el desarrollo conjunto de materiales, proyectos y actividades puede incidir, en distinta medida, en las relaciones interprofesionales y los aprendizajes docentes, la resolución conjunta de problemas parece impactar fuertemente en la tarea docente. En efecto, Senge (2002) reconocía en la resolución de problemas un gran potencial de aprendizaje para los miembros de una organización, en tanto mejora su adaptabilidad al entorno y genera un marco de discusión creativa cuyos frutos terminan incorporándose a la cultura institucional.

La coordinación se asoma, pues, como una de las modalidades más débiles de trabajo colaborativo: no requiere de valores compartidos, no genera relaciones de interdependencia y no fomenta necesariamente el aprendizaje. El desarrollo de proyectos interdisciplinarios y la resolución conjunta de problemas, en cambio, demandan una fuerte interdependencia sobre la base de valores compartidos, a la vez que ofrecen intercambios con una gran potencialidad para generar nuevos aprendizajes. 


\section{CONCLUSIONES}

No es fácil desarrollar la colaboración entre docentes, y aun cuando los profesores trabajen de forma colectiva esto no siempre supone intercambios profundos, sustanciales y transparentes sobre los fines de la educación, los dilemas de la práctica o las demandas de aprendizaje. En una obra clásica, Sarason (1982) explica que hace falta mucha observación, discusión y comunicación en las culturas escolares para reflexionar sobre los comportamientos profesionales y, si procede, cambiarlos. Nuestra categorización de las modalidades de colaboración docente pretende ofrecer un marco de análisis sobre los formatos de la colegialidad y los tipos de intercambios que suponen, para intentar estimar, aunque sea parcialmente, su potencialidad a la hora de generar modificaciones en las prácticas de enseñanza.

Hemos constatado que la colaboración entre el profesorado adopta distintos matices y está al servicio de distintos propósitos. Como dice Little (2002), puede impulsar prácticas innovadoras o perpetuar estructuras conservadoras. La investigación sobre el trabajo colaborativo resulta entonces estratégica de cara al cambio en educación: entender qué hacen los profesores cuando se reúnen y qué impacto tiene ello en su enseñanza nos permitirá comprender por qué algunas escuelas consiguen mejorar y otras no tanto, sabiendo que las prácticas colaborativas que maximizan el aprendizaje docente guardan mayor relación con la mejora escolar (Bolam y otros, 2005). Se requieren entonces de nuevos estudios que profundicen nuestra comprensión acerca del desarrollo de una verdadera colaboración para el aprendizaje y la mejora escolar.

La colaboración puede resultar estratégica para el docente en la medida en la que lo nutra de nuevas herramientas pedagógicas ante los problemas concretos de su práctica, reforzando así su autonomía y capacidad de decisión. Por ello los docentes necesitan trabajar en entornos colaborativos donde abunde el apoyo mutuo, las responsabilidades compartidas y la reflexión sistemática. No es un paso suficiente pero sin duda es un camino necesario para estimular la capacidad de cambio e innovación en las escuelas. 


\section{REFERENCIAS BIBLIOGRÁFICAS}

Bolam, R., McMahon, A., Stoll, L., Thomas, S. \& Wallace, M. (2005). Creating and sustaining professional learning communities. Research Report Number 637. Londres: General Teaching Council for England.

Butler, D. L. \& Schnellert, L. (2012). Collaborative inquiry in teacher professional development. Teaching and Teacher Education, 28(8), 1206-1220.

Crawford, M. (2012). Solo and distributed leadership: Definitions and dilemmas. Educational Management Administration \& Leadership, 40(5), 610-620.

Day, Ch. (2005). Formar docentes: cómo, cuándo y en qué condiciones aprende el profesorado. Madrid: Narcea.

Donmoyer, R., Yennie-Donmoyer, J. \& Galloway, F. (2012). The search for connections across principal preparation, principal performance, and student achievement in an exemplary principal preparation program. Journal of Research on Leadership Education, 7(1), 5-43.

DuFour, R., DuFour, R., Eaker, R. \& Many, Th. (2010). Learning by doing. A Handbook for Professional Learning Communities at Work. Bloomington, IN: Solution Tree Press.

Duke, D. L. (2006). What we know about improving low-performing schools. Phi Delta Kappan, 87(10), 728-734.

Escudero Muñoz, J. M. (2009). Comunidades docentes de aprendizaje, formación del profesorado y mejora de la Educación. Ágora para la Educación Física y el Deporte, 10, 7-31.

Gairín, J. (2000). Cambio de cultura y organizaciones que aprenden. Educar, 27, 31-85.
Glaser, B. y Strauss, A. (1967). The Discovery of Grounded Theory: Strategies for Qualitative Research. Chicago, IL: Aldine Publishing Company

Goddard, R., Hoy, W. \& Hoy, A. (2000). Collective teacher efficacy: Its meaning and meassure, and impact on student achievement. American Educational Research Journal, 37(2), 479-507.

Grossman, P., Wineburg, S. \& Woolworth, S. (2001). Toward a theory of teacher community. Teachers College Record, 103(6), 942-1012.

Hargreaves, A. \& Shirley, D. (2012). The Global Fourth Way. The Quest for Educational Excellent. Thousand Oaks, CA: Corwin Press.

Hopkins, D. (2010). A Teacher's guide to Classroom Research. Maidenhead: Open University Press.

Hord, S. y Hirsh, S. (2008). Making the promise a reality. En A. Blankstein, P. Houston y R. Cole (Eds.), Sustaining Professional Learning Communities (pp. 23-40). Thousand Oaks, CA: Corwin Press.

Horn, I. \& Little, J. (2010). Attending to problems of practice: Routines and resources for professional learning in teachers' workplace interactions. American Educational Research Journal, 47(1), 181-217.

Huberman, M. (1993). The model of the independent artisan in teachers' professional relationships. En J. Little y M. McLaughlin (Eds.), Teachers' Work: Individuals, Colleagues and Contexts (pp.11-50). Nueva York: Teachers College Press.

Huffman, J. \& Hipp, K. (2003). Reculturing Schools as Professional Learning Communities. Lanham: Scarecrow Education. 
Jäppinen, A., Leclerc, M. \& Tubin, D. (2015). Collaborativeness as the core of professional learning communities beyond culture and context: evidence from Canada, Finland, and Israel. School Effectiveness and School Improvement: An International Journal of Research, Policy and Practice, [online] DOI:10.1080/09243453.2015.1067235

King, M. y Bouchard, K. (2011). The capacity to build organizational capacity in schools. Journal of Educational Administration, 49(6), 653-669.

Lavié Martínez, J. M. (2004a). Microcontextos para la colaboración docente: el caso de los equipos de ciclo. Revista de Educación, 335, 345-370.

Lavié Martínez, J. M. (2004b). Individualismo y privacidad en la cultura docente: revisando los argumentos. Revista Española de Pedagogía, 229, 439-454.

Lavié Martínez, J. M. (2006). Academic Discourses on School-Based Teacher Collaboration: Revisiting the Arguments. Educational Administration Quarterly, 42(5), 773-805.

Little, J. (1990). The persistence of privacy: autonomy and initiative in teacher's professional relations. Teachers College Record, 91(4), 509- 535.

Little, J. (2002). Locating learning in teachers' communities of practice: opening up problems of analysis in records of everyday work. Teaching and Teacher Education, 18(8), 917- 946.

Little, J. \& Horn, I. (2007). 'Normalizing' problems of practice: converting routine conversation into a resource for learning in professional communities. En L. Stoll \& K. S. Louis (Eds.), Professional Learning Communities: Divergence, Depth and Dilemmas (pp. 79-92). Columbus, OH: Open University Press.
Lortie, D. (1975). Schoolteacher: A sociological study. Chicago, IL: The University of Chicago Press.

Martin, P. C. (2014). Stumped by Student Needs: Factors in Developing Effective Teacher Collaboration. Electronic Journal for Inclusive Education, 3(2).

McLaughlin, M. \& Talbert, J. (2006). Building School-Based Teacher Learning Communities. Professional strategies to improve student achievement. Nueva York: Teacher's College Press.

Muijs, D. \& Rumyantseva, N. (2014). Coopetition in education: Collaborating in a competitive environment, Journal of Educational Change, 15(1), $1-18$.

Rosenholtz, S. (1989). Teachers Workplace: The Social Organization of Schools. White Planis, NY: Longman.

Sarason, S. (1982). Culture of the school and the problem of change. Boston: Allyn \& Bacon.

Sebastian, J. \& Allensworth, E. (2012). The influence of principal leadership on classroom instruction and student learning: A study of mediated pathways to learning. Educational Administration Quarterly, 48(4), 626-663.

Senge, P. (2002). La Quinta Disciplina: Escuelas que Aprenden. Bogotá: Norma.

Silins, H. \& Mulford, W. (2004). Schools as Learning Organizations. Effects on Teacher Leadership and Student Outcomes. School Effectiveness and School Improvement, 15(3-4), 443-466.

Smyth, J. (2001). Critical politics of teachers work: An Australian perspective. Nueva York: Peter Lang. 
Szczesiul, S. (2014). The [un]spoken challenges of administrator collaboration: An exploration of one district leadership team's use of protocols to promote reflection and shared theories of action. Journal of Educational Change, 15(4), 411-442.

Szczesiul, S. \& Huizenga, J. (2014). The burden of leadership: Exploring the principal's role in teacher collaboration. Improving Schools, 17(2), 176-191.
Thoonen, E., Sleegers, P., Oort, F. \& Peetsma, T. (2012). Building schoolwide capacity for improvement: The role of leadership, school organizational conditions, and teacher factors. School Effectiveness and School Improvement, 23(4), 441-460.

Vescio, V., Ross, D. \& Adams, A. (2008). A review of research on the impact of profesional learning communities on teaching practice and student learning. Teaching and teacher education, 24(1), 80-91. 


\section{PERFIL ACADÉMICO Y PROFESIONAL DE LOS AUTORES}

Gabriela Krichesky. Doctora en Educación (Universidad Autónoma de Madrid). Profesora del Área de Educación de la Universidad Torcuato Di Tella. Se ha desempeñado como profesora en la Universidad Autónoma de Madrid, la Universidad de Córdoba y la Universidad Carlos III de Madrid. Sus principales áreas de investigación son la Mejora Escolar y el Liderazgo Educativo. Es miembro del Grupo de Investigación «Cambio Educativo para la Justicia Social» y coordina programas de capacitación para directivos escolares.

F. Javier Murillo. Profesor Titular de MIDE en la Universidad Autónoma de Madrid. Coordinador del Doctorado en Educación y del Grupo de Investigación «Cambio Educativo para la Justicia Social». Director de REICE. Revista Iberoamericana sobre Calidad, Eficacia y Cambio en Educación, de la Revista Iberoamericana de Evaluación Educativa y de la Revista Internacional de Educación para la Justicia Social (RIEJS). Fue Coordinador General del Laboratorio Latinoamericano de Evaluación de la Calidad de la Educación (LLECE) de la UNESCO.

Dirección de los autores: Gabriela J. Krichesky

Universidad Torcuato Di Tella

Av. Figueroa Alcorta, 7350

(CP1428) Buenos Aires

Argentina

E-mail: gkrichesky@utdt.edu

F. Javier Murillo

Universidad Autónoma de Madrid

$\mathrm{C} /$ Tomas y Valiente, 3

28049 Madrid

España

E-mail: javier.murillo@uam.es

Fecha recepción del artículo: 27. Julio. 2015

Fecha modificación del artículo: 28. Octubre. 2015

Fecha aceptación del artículo: 21. Noviembre. 2015

Fecha revisión para publicación: 31. Mayo. 2017 
